\title{
An Alternative Treatment for Vaginal Cuff Wart: a Case Report
}

\author{
Victoria Psomiadou, Christos lavazzo*, Athanasios Douligeris, Alexandros Fotiou, \\ Anastasia Prodromidou, Nikolaos Blontzos, Evgenia Karavioti, George Vorgias
}

\begin{abstract}
Human papillomavirus (HPV) has been directly related to acuminate warts and cervical cancer, the second most common neoplasia among women. Given the lack of treatment against the virus itself, many medications have been utilised, mainly aiming in modifying the host's immunological response. We present the case of a 54 years old postmenopausal patient with a history of vaginal cuff wart and HPV persistence that we managed in our clinic for 6 months with a mix of curcumin, aloe vera, amla and other natural ingredients. As the patient was found to be intolerant to imiquimod (one of the most common conservative methods of treatment) we attempted the use of curcumin, which was applied to the area of the wart three times per week for 6 months. Both clinical and colposcopical improvement was noted in regular clinic visits with regression of the lesion. The outcome of this case encourages our view that curcumin should be considered as a significant treatment modality against HPV infection and acuminate warts.
\end{abstract}

\section{KEYWORDS}

HPV; vaginal cuff wart; curcumin; Indian Grapefruit (AMLA); aloe vera; Docosanol; lactic acid; CM- $\beta$ glucans; SiloffGyn

\section{AUTHOR AFFILIATIONS}

Department of Gynecologic Oncology, Metaxa Memorial Cancer Hospital, Piraeus, Greece

* Corresponding author: Nosokomeio Metaxa, Gynaecological Oncology, 51, Botassi Str, Piraeus, GR 18537;

e-mail: christosiavazzo@hotmail.com

Received: 17 April 2019

Accepted: 4 December 2019

Published online: 18 May 2020

Acta Medica (Hradec Králové) 2020; 63(1): 49-51

https://doi.org/10.14712/18059694.2020.15

(c) 2020 The Authors. This is an open-access article distributed under the terms of the Creative Commons Attribution License (http://creativecommons.org/licenses/by/4.0), which permits unrestricted use, distribution, and reproduction in any medium, provided the original author and source are credited. 


\section{INTRODUCTION}

Cervical cancer is the 4th most common cancer among women worldwide, with an estimated 527,624 new cases and 265,672 deaths in 2012 (1). HPV is recognized as a well-established causative factor, mainly through the work of Harald zur Hausen, who also later identified the two most oncogenic types of the virus, 16 and 18 (2). Nowadays there is upcoming conclusive evidence with respect to carcinogenicity of HPV in other anogenital cancers (anus, vulva, vagina and penis) as well as head and neck cancers (3).

Primary HPV infection usually occurs early in life and it is asymptomatic in most cases. Genital warts are the visible manifestation of the infection, typically caused by HPV types 6 and 11, which rarely are associated with invasive squamous cell carcinoma (4). Patients with condylomata (genital warts) can present with burning, itching, bleeding, and pain as well as psychological anxiety and embarrassment (5). The disease is estimated to lead to high morbidity and significant healthcare costs, since the lesions typically recur even after different ablative (electrocautery, liquid nitrogen, and laser therapy) techniques or surgical excision. Specifically, the recurrence rate with each technique reach $20-40 \%$ for cryotherapy, $15 \%$ for imiquimod, $5-50 \%$ for laser treatment, $5-30 \%$ for podofilox and $20-65 \%$ for podophyllin resin (6).

Currently many natural plant origin compounds have been identified as promising sources of drugs for treatment and prevention against recurrence of genital warts, with podofilox and imiquimod being the most recommended (7). Another non-invasive treatment agent is curcumin, a topical immune response modifier, isolated from the root of Curcuma longa. Curcumin, introduced as a safe and effective treatment for HPV-associated genital warts, has not been found to achieve its optimum therapeutic outcome, mainly because of its low solubility and poor bioavailability. Lately, it has been developed as a therapeutic drug through alterations in formulation properties and improvement of delivery systems (8).

We present a case of a vaginal cuff wart diagnosed and managed in our clinic with a mix of curcumin, aloe vera, amla and other natural ingredients. Our aim is to discuss an alternative option in the treatment of acute warts that persist or recur after other methods of therapy.

\section{CASE REPORT}

A 54-year-old woman, with a HGSIL Pap smear was referred to our clinic. The patient has a THBO medical history due to uterus fibroids ten years ago. Clinical examination and colposcopy revealed a vaginal cuff wart and histopathology confirmed the HPV infection. The patient was treated with ALDARA 5\% cream, a regimen of imiquimod produced by Meda AB in Sweden, which was applied three times weekly. However, her clinical situation worsened after two cycles of therapy as she presented with symptoms of intense intolerance of the medicament, including burning and itchiness of the affected area. Administration of imiquimod was discontinued for the following two months. The patient was then reevaluated and an alternative treatment with a mixture of curcumin, Indian Grapefruit (AMLA), aloe vera, Docosanol, lactic acid and CM- $\beta$ glucans was applied. The vaginal cream SiloffGyn, produced by Heremco Pharmaceuticals in Athens, was locally applied at the wart, daily for one week and then three times a week. Three months later the patient's clinical and colposcopy findings improved; both Pap smear result and colposcopy were negative. Two years later the patient has no clinical or laboratory suspicion of recurrence.

\section{DISCUSSION}

HPVs belong to the family of papilloma viruses, usually infecting exclusively skin and mucosal surfaces of the mouth, the anal, the female genitalia, and the epithelium of the endometrium resulting in cell proliferation and proliferative, precancerous, but also cancerous lesions. To date, 189 types of papillomaviruses have been identified, 120 of which infect humans (9).

HPV infection is the most common sexually transmitted disease in the U.S, affecting almost $1 \%$ of the sexually active population (10). Warts or condylomata are etiologically associated with some HPV types, mainly low risk types 6 or 11, but not exclusively. In contrast with the cervical intraepithelial neoplasias, which are silent, warts are usually noticed by the patient herself/himself and they vary from flat papules to large, cauliflower-like lesions. Diagnosis is clinical, but atypical lesions should be confirmed by histology. Therapy ranges between surgical (excision, electrosurgery, cryotherapy) and conservative. The latter depends on the topical application of various medicaments such as imiquimod, podofilox, podophyllin, bichloroacetic acid, and trichloroacetic acid. The cure rates are of each method are estimated to reach $30-50 \%$, $45-80 \%, 30-80 \%$ and $50-80 \%$ respectively (6).

Derived from the perennial herb Curcuma longa (turmeric) curcumin is a polyphenol of plant origin known since many years as traditional Indian medicine. Turmeric

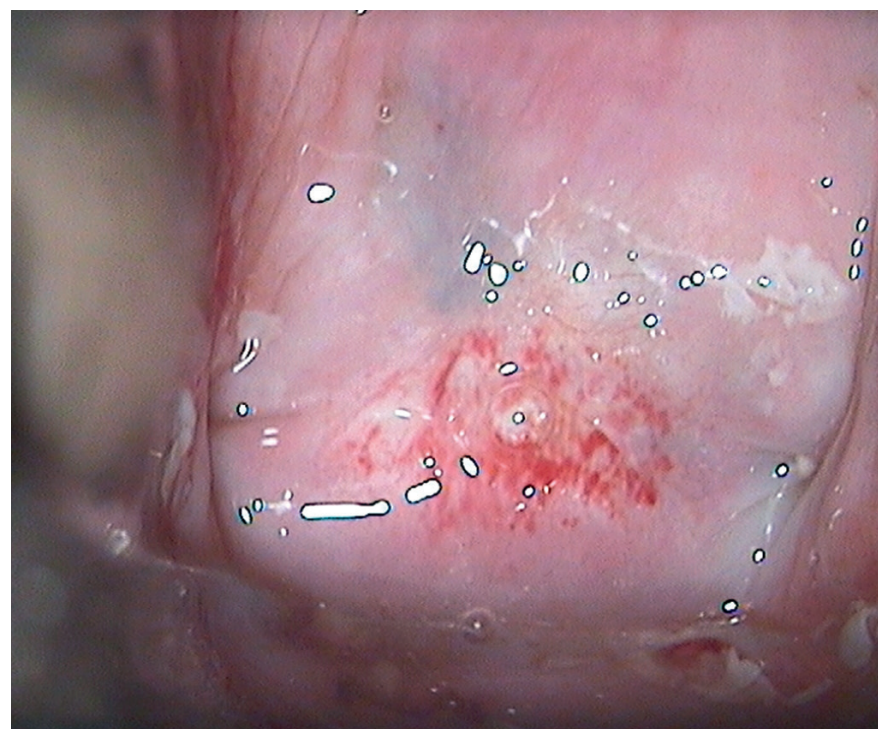

Fig. 1 Vaginal cuff wart before treatment. 
was introduced into Europe in the 13th century by Marco Polo and surprisingly, since 1937, when an early study was published in The Lancet by Oppenheimer, it is in the last 15 years that it has gained increasing popularity and it has become the subject of many studies (8).

In the last decades, curcumin has been found to mediate in various cell signaling molecules and this way to downregulate inflammation mediators, cytokines, interleukins and enzymes, gaining anti-inflammatory effects and therapeutic potential against a wide range of pathologic conditions, such as many types of cancer, inflammatory bowel disease, osteoarthritis, H. Pylori infection, psoriasis, acute coronary syndrome, atherosclerosis, type 2 diabetes, renal transplantation and $\beta$-Thalassemia (11-13).

In the context of the aforementioned multiple properties, curcumin is considered cytotoxic against cervical cancer cells and has been found to downregulate the expression of HPV oncoproteins. Importantly, with regards to its safety, turmeric has been established to be safe and well-tolerated by human trials and is Generally Recognized As Safe (GRAS) by the US FDA (14). Interestingly, Debata et al, recently developed a curcumin-based vaginal cream that eradicates HPV positive cancer cells without affecting the healthy tissues (15).

\section{CONCLUSIONS}

As far as we know, most of the curcumin-related studies have highlighted its potential to clear HPV infection, and consequently the intraepithelial precancerous lesions it induces. Literature review about condylomata treatment with curcumin was poor but encouraging. The patient applied vaginally curcumin 2 times a week without further anti HPV treatment for 6 months and for 18 months now she has remained recurrent-free. Repeat cytology confirmed the recession of the wart. To the best of our knowledge, this is the first report in which curcumin has demonstrated an objective response in conventional treatment of a vaginal cuff condyloma.

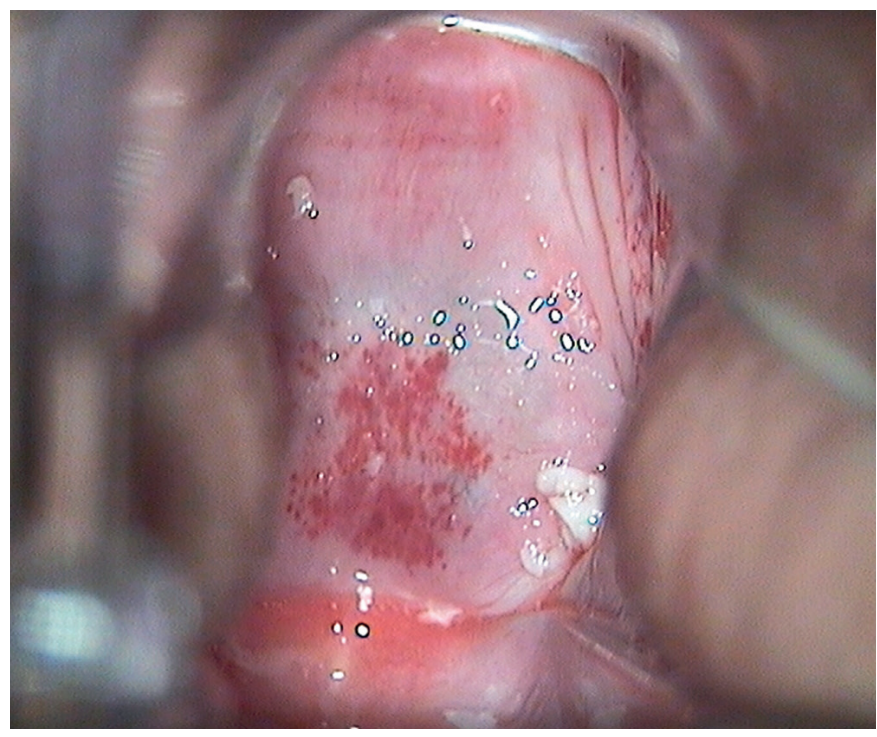

Fig. 2 Vaginal cuff wart after treatment with imiquimode.

\section{LIST OF ABBREVIATIONS}

THBO - Total Hysterectomy with Bilateral Oophorectomy

\section{REFERENCES}

1. Bruni L AG, Serrano B, Mena M, et al. ICO/IARC Information Centre on HPV and Cancer (HPV Information Centre). Human Papillomavirus and Related Diseases in the World. Summary Report 17 June 2019. [15 November 2019].

2. zur Hausen H. Papillomaviruses in the causation of human cancers a brief historical account. Virology 2009; 384(2): 260-5.

3. de Martel C, Ferlay J, Franceschi S, et al. Global burden of cancers attributable to infections in 2008: a review and synthetic analysis. Lancet Oncology 2012; 13(6): 607-15.

4. Burd EM. Human papillomavirus and cervical cancer. Clin Microbiol Rev 2003; 16(1): 1-17.

5. Lopaschuk CC. New approach to managing genital warts. Can Fam Physician 2013; 59(7): 731-6.

6. Kodner CM, Nasraty S. Management of genital warts. Am Fam Physician 2004; 70(12): 2335-42.

7. Komericki P, Akkilic-Materna M, Strimitzer T, Aberer W. Efficacy and safety of imiquimod versus podophyllotoxin in the treatment of anogenital warts. Sex Transm Dis 2011; 38(3): 216-8.

8. Basnet P, Skalko-Basnet N. Curcumin: an anti-inflammatory molecule from a curry spice on the path to cancer treatment. Molecules $2011 ; 16(6): 4567-98$

9. Braaten KP, Laufer MR. Human Papillomavirus (HPV), HPV-Related Disease, and the HPV Vaccine. Rev Obstet Gynecol 2008; 1(1): 2-10.

10. McQuillan G K-MD, Markowitz LE, Unger ER, Paulose-Ram R. Prevalence of HPV in adults aged 18-69: United States, 2011-2014. NCHS data brief, no 280 . Hyattsville, MD. National Center for Health Statistics. 2017.

11. Gupta SC, Patchva S, Aggarwal BB. Therapeutic roles of curcumin: lessons learned from clinical trials. AAPS J 2013; 15(1): 195-218.

12. Teymouri M, Pirro M, Johnston TP, Sahebkar A. Curcumin as a multifaceted compound against human papilloma virus infection and cervical cancers: A review of chemistry, cellular, molecular, and preclinical features. Biofactors 2017; 43(3): 331-46.

13. Shishodia G, Verma G, Srivastava Y, Mehrotra R, Das BC, Bharti AC. Deregulation of microRNAs Let-7a and miR-21 mediate aberrant STAT3 signaling during human papillomavirus-induced cervical carcinogenesis: role of E6 oncoprotein. BMC Cancer 2014; 14: 996.

14. Karnes JB, Usatine RP. Management of external genital warts. Am Fam Physician 2014; 90(5):312-8.

15. Debata PR, Castellanos MR, Fata JE, et al. A novel curcumin-based vaginal cream Vacurin selectively eliminates apposed human cervical cancer cells. Gynecologic Oncology 2013; 129(1): 145-53.

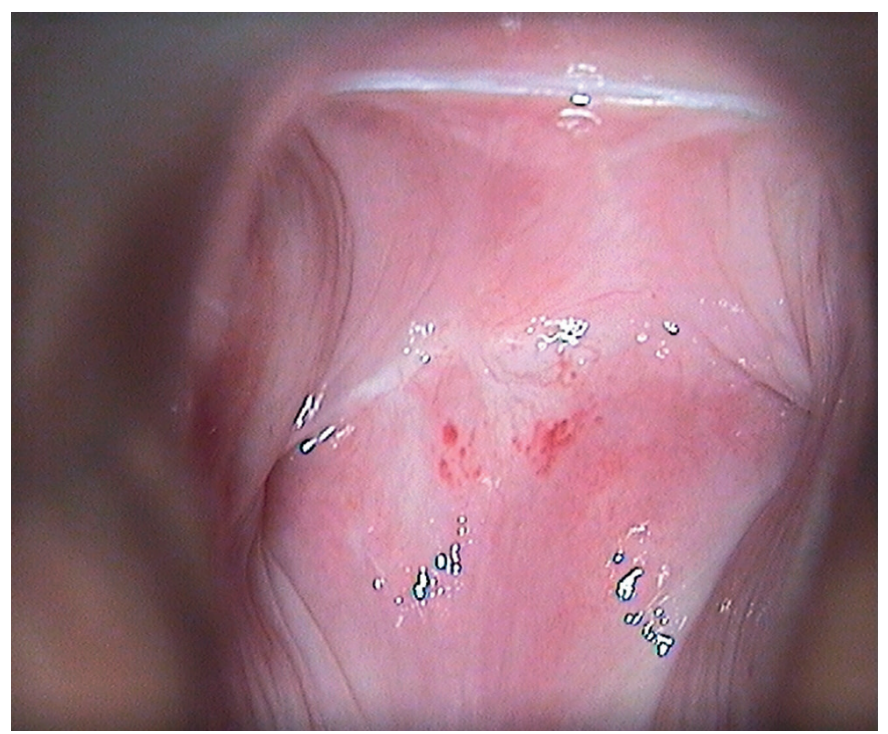

Fig. 3 Vaginal cuff wart after treatment with SiloffGyn. 